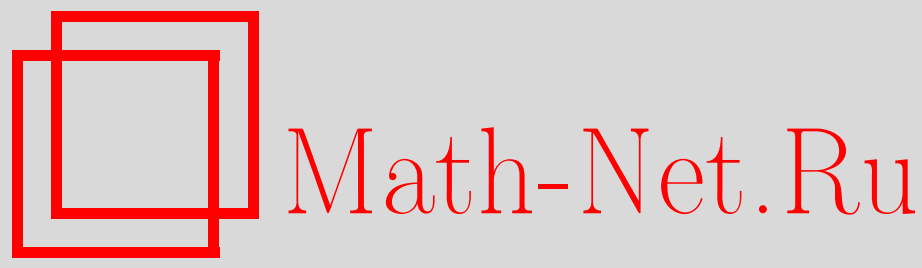

W. Narkiewicz, On a Class of Monic Binomials, Совр. пробл. матем., 2012, выпуск 16, 76-81

DOI: https://doi.org/10.4213/spm36

Использование Общероссийского математического портала Math-Net.Ru подразумевает, что вы прочитали и согласны с пользовательским соглашением http://www.mathnet.ru/rus/agreement

Параметры загрузки:

IP : 54.224 .60 .19

26 апреля 2023 г., 18:16:20

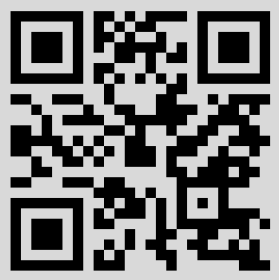




\title{
On a Class of Monic Binomials
}

\author{
W. Narkiewicz \\ Institute of Mathematics, Wroctaw University, Poland
}

Dedicated to the memory of Professor A. A. Karatsuba

1. If $f(X)$ is a polynomial with coefficients in a field $K$, then the sequence $a_{1}, a_{2}, \ldots, a_{k}$ of distinct elements of $K$ is called an $f$-cycle of length $k$, if $f\left(a_{k}\right)=a_{1}$ and for $j=1,2, \ldots, k-1$ one has $f\left(a_{j}\right)=a_{j+1}$. The truth of a conjecture of Morton and Silverman [1] would imply that if $K$ is an algebraic number field of degree $N$, then the length of any such cycle is bounded by a constant depending only on $N$ and the degree of the polynomial $f$. It was shown in [2] that if $f(X)=X^{n}+c$ is a binomial of an odd prime-power degree $n=p^{m}$, and $K$ is an algebraic number field of degree $N$ not containing the $p$ th roots of unity, then the lengths of cycles of $F$ in $K$ are bounded by a certain constant $c=c(K, p)$, and in the case when the prime $p$ exceeds $2^{N}$, then this bound depends only on $N$. The purpose of this note is to show that a modification of the argument in [2] leads to a similar result for polynomials of the form $X^{n}+c \in K[X]$ when $n$ is odd and for every prime divisor $q$ of $n$ one has $q-1 \nmid N$. Actually we establish this for a larger class of fields $K$ (see Theorem 2 in Sect. 3). Note that it suffices to consider the case of totally complex fields $K$ as in a real field the polynomial $X^{n}+c$ is increasing hence cannot have cycles of length $\geqslant 2$.

2. We start with an auxiliary result established earlier ([2]) in the case when $n$ is a prime power. Recall that a monic polynomial has bad reduction at a prime ideal $\mathfrak{p}$ if at least one of its coefficients is not $\mathfrak{p}$-integral.

THEOREM 1. Let $K$ be an algebraic number field, $\mathbb{Z}_{K}$ its ring of integers and $f(X)=X^{n}+c$ with odd $n$ and $c \in K \backslash \mathbb{Z}_{K}$. If $f$ has in $K$ a cycle of length $\geqslant 3$ and $\mathfrak{p}$ is a prime ideal of $\mathbb{Z}_{K}$ at which $f$ has bad reduction, then either $\mathfrak{p}$ divides $n \mathbb{Z}_{K}$ or there exists a prime divisor $q$ of $n$ satisfying

$$
N(\mathfrak{p}) \equiv 1(\bmod q)
$$

To prove it we shall use the following auxiliary result proved in [2, Lemmas 2, 3], which generalizes slightly the results obtained in [3, Corollary 4] and [4, Corollary 6.7].

Lemma 1. Let $f(X)=X^{n}+c$ with $n \geqslant 2$ and $c \in K \backslash \mathbb{Z}_{K}$, and assume that $r_{1} \mapsto \cdots \mapsto r_{k} \mapsto r_{1}$ is an $f$-cycle of length $k \geqslant 3$ lying in $K$.

(i) Put $I_{0}=c \mathbb{Z}_{K}, I_{j}=r_{j} \mathbb{Z}_{K}, j=1,2, \ldots, k$, and write

$$
I_{j}=A_{j} B_{j}^{-1}, \quad j=0,1, \ldots, k,
$$

where $A_{0}, A_{1}, \ldots, A_{k}, B_{0}, B_{1}, \ldots, B_{k}$ are ideals of $\mathbb{Z}_{K}$ satisfying $\left(A_{j}, B_{j}\right)=1$ for $j=0,1, \ldots, k$. Then the ideal $B_{0}$ is an $n$th power, say $B_{0}=B^{n}$, and for $j=1,2, \ldots, k$ one has $B_{j}=B$.

(ii) There is a class $\mathbb{X}$ in the class-group of ideals of $\mathbb{Z}_{K}$ such that if the ideal $I$ lies in $\mathbb{X}$ and is prime to $B$, then there exist $a, b, N_{1}, \ldots, N_{k} \in \mathbb{Z}_{K}$ such that

$$
c=\frac{a}{b^{n}}, \quad r_{j}=\frac{N_{j}}{b}, \quad\left(a \mathbb{Z}_{K}, b^{n} \mathbb{Z}_{K}\right)=I^{n}, \quad\left(N_{j} \mathbb{Z}_{K}, b \mathbb{Z}_{K}\right)=I, \quad j=1,2, \ldots, k .
$$

If we extend the sequence $N_{j}$ by periodicity, putting $N_{j+k}=N_{j}$ for $j \geqslant 1$, then one has

$$
\left(N_{j} \mathbb{Z}_{K}, B\right)=1
$$


and

$$
\prod_{i=1}^{k} \frac{N_{i+1}^{n}-N_{i}^{n}}{N_{i+1}-N_{i}}=b^{k(n-1)} .
$$

We need also the following well-known result.

LEMMA 2. If $a \neq b$ are non-zero elements of $\mathbb{Z}_{K}$ and $n \geqslant 2$, then for every prime ideal $\mathfrak{p}$ of $\mathbb{Z}_{K}$ containing $\left(a^{n}-b^{n}\right) /(a-b)$ either $\mathfrak{p}$ divides both $a \mathbb{Z}_{K}$ and $b \mathbb{Z}_{K}$, or $\mathfrak{p} \mid n \mathbb{Z}_{K}$ and $a \equiv b(\bmod \mathfrak{p})$, or, finally, for some prime divisor $q$ of $n$ one has $N \mathfrak{p} \equiv 1(\bmod q)$.

Proof of Theorem 1. Assume that the polynomial $f(X)=X^{n}+c$ has a cycle of length $k \geqslant 3$. Let $N_{1}, \ldots, N_{k}$ and $B$ be as in Lemma 1 . Since $c \notin \mathbb{Z}_{K}$ we have $B \neq \mathbb{Z}_{K}$. If $\mathfrak{p}$ is a prime ideal divisor of $B$, then $b \in \mathfrak{p}$ and the equality (3) implies that for some $i$ we have

$$
\frac{N_{i+1}^{n}-N_{i}^{n}}{N_{i+1}-N_{i}} \in \mathfrak{p} .
$$

Lemma 2 implies now that either $\mathfrak{p}$ divides both $N_{i} \mathbb{Z}_{K}$ and $N_{i+1} \mathbb{Z}_{K}$, or $\mathfrak{p} \mid n \mathbb{Z}_{K}$ and $N_{i} \equiv$ $N_{i+1}(\bmod \mathfrak{p})$, or for some prime divisor $q$ of $n$ one has $N(\mathfrak{p}) \equiv 1(\bmod q)$, but the first possibility is excluded by (2).

3. Let $n \geqslant 3$ be an odd integer and denote by $\mathcal{X}_{n}$ the family of algebraic number fields $K$ of finite degree such that almost all (i.e., all except finitely many) prime ideals of the first degree in the ring $\mathbb{Z}_{K}$ of integers of $K$ have their norms congruent to unity $\bmod q$ for a prime $q$ dividing $n$. Note that the family $\mathcal{X}_{n}$ depends only on the set of prime divisors of $n$.

Now we state our results.

TheOREM 2. Let $n$ be an odd integer, let $K$ be an algebraic number field of degree $N$, not belonging to the family $\mathcal{X}_{n}$ and let $f(X)=X^{n}+c$ with $c \in K \backslash \mathbb{Z}_{K}$.

(i) There exists a constant $B=B(K, n)$ depending only on $K$ and $n$ such that the length of a cycle of $f(X)$ lying in $K$ does not exceed $B(k, n)$.

(ii) If all prime divisors $q$ of $n$ satisfy $q>2^{N}$, then $B(K, n)$ depends only on the degree $N$ of $K$. If 2 splits in $K$, then $B(K, n) \leqslant 2$.

COROllary 1. If $n$ is odd and $K \notin \mathcal{X}_{n}$ is an Abelian extension of the rationals of conductor $F$, then for every $\epsilon>0$ one has $B(K, n) \ll_{\epsilon}(F M)^{L+1+\epsilon}$, where L denotes the Linnik constant and $M$ is the product of prime divisors of $n$.

COROLlaRY 2. If $n$ is a product of two odd prime-powers, $K$ is an algebraic number field of degree $N$, and for primes $q \mid n$ that field does not contain roots of unity of order $q$ and $f(X)=$ $x^{n}+c$ with $c \in K \backslash \mathbb{Z}_{K}$, then the lengths of $f$-cycles in $K$ are bounded by a value depending only on $K$ and $n$.

Corollary 3. If $n$ is odd, $f(X)=X^{n}+c$ with $c \in K \backslash \mathbb{Z}_{K}$, where $K$ is an algebraic number field of degree $N$ with trivial class-group, and for primes $q \mid n$ that field does not contain roots of unity of order $q$, then the lengths of $f$-cycles in $K$ are bounded by a value depending only on $K$ and $n$.

COROLlary 4. If $n$ is odd and square-free, $f(X)=X^{n}+c$ with $c \in K \backslash \mathbb{Z}_{K}$, where $K \notin \mathcal{X}_{n}$ is a Galois extension of the rationals of degree $N$ and for prime divisors $q$ of $n$ we have $q-1 \nmid N$, then the lengths of $f$-cycles in $K$ are bounded by a value depending only on $K$ and $n$.

4. To simplify the check whether a given field $K$ lies in $\mathcal{X}_{n}$ we provide first a few simple assertions about the family $\mathcal{X}_{n}$. We shall henceforth assume that every non-zero element of $K$ has a positive norm. This holds in particular in totally complex fields. 
LEMMA 3. Let $K$ be an algebraic number field, $n$ a positive rational integer, $\mathfrak{f}=n \mathbb{Z}_{K}$ and denote by $H_{n}$ the class-group mod $\mathfrak{f}$. If $I, J$ are two ideals lying in the same class in $H_{n}$, then $N(I) \equiv N(J)(\bmod n)$.

Proof. The assumption implies the existence of $a, b \in \mathbb{Z}_{K}$ with $a \equiv b(\bmod \mathfrak{f}),\left(a b \mathbb{Z}_{K}, \mathfrak{f}\right)=1$ and $a I=b J$. Since $\mathfrak{f}$ has a rational generator we have

$$
N(a) \equiv N(b)(\bmod n),
$$

and since norms of $a, b$ are positive we get

$$
N(a) N(I)=N(a I)=N(b J)=N(b) N(J)
$$

and $N(I) \equiv N(J)(\bmod n)$.

Corollary 5. If $K \in \mathcal{X}_{n}, I$ is an ideal of $\mathbb{Z}_{K}$, prime to $n$, then for some prime divisor $q$ of $n$ one has $N(I) \equiv 1(\bmod q)$.

Proof. Recall that in every ideal class in $H_{n}$ there are infinitely many prime ideals of first degree and apply the lemma.

By $\mathcal{Y}_{n}$ we shall denote the family of all fields $K$ containing $Q\left(\zeta_{q}\right)$ for some prime divisor $q$ of $n$. One sees easily that one has always $\mathcal{Y}_{n} \subset \mathcal{X}_{n}$ and it follows from an old theorem of Bauer [5] that if $n$ is a prime, then $\mathcal{Y}_{n}=\mathcal{X}_{n}$. It is not difficult to show that the same is true if $n$ is a product of two primes (see Theorem 3 (i) below), but the following example shows that $\mathcal{X}_{n}$ may contain fields without any roots of unity $\neq \pm 1$.

Let $K=Q\left(\zeta_{n}\right)$ with $n=105$ and let $k$ be its subfield corresponding to the subgroup $H=$ $\{1,29,34,41\}$ of the Galois group $G(n)=C_{12} \oplus C_{2}^{2}$ of $K / Q$.

The extension $k / Q$ is of degree 12 , has a cyclic Galois group and its discriminant equals $3^{6} \cdot 5^{9} \cdot 7^{10}$. If $p$ is a rational prime splitting in $k$, then $p(\bmod 105) \in H$, hence $p \equiv 1(\bmod q)$ for some $q \in\{3,5,7\}$, and since every ideal-class $\bmod 105 \mathbb{Z}_{k}$ contains infinitely many such primes $p$ the Corollary 5 implies $k \in \mathcal{X}_{105}$.

5. We show now that in many cases a field contained in $\mathcal{X}_{n}$ must contain a root of unity $\zeta_{q}$, $q$ being a prime divisor of $n$. Recall that we work under the standing assumption that $K$ does not contain elements of negative norm. We need a simple lemma in which we denote by $\omega(m)$ the number of distinct prime divisors of $m$.

Lemma 4. Let $n$ be an integer with $\omega(n) \geqslant 3$. If $K \in \mathcal{X}_{n}$ and the class-number of $K$ equals 1 , then there is a divisor $d$ of $n$ with $\omega(d)<\omega(n)$ such that $K \in \mathcal{X}_{d}$.

Proof. Put $s=\omega(n)$, let $n=\prod_{j=1}^{s} p_{j}^{\alpha_{j}}$ with prime $p_{j}$ and $a_{j} \geqslant 1$, and put $\mathfrak{f}=n \mathbb{Z}_{K}$. If the assertion would fail, then by the Corollary 5 there would exist an ideal $I$ in $\mathbb{Z}_{K}$ with $(I, \mathfrak{f})=1$ and

$$
N(I) \equiv r_{j}\left(\bmod p_{j}\right), \quad j=1,2, \ldots, s,
$$

where $r_{1}=1$ and $r_{j} \not \equiv 0,1\left(\bmod p_{j}\right)$ for $j=2,3, \ldots, s$. Indeed, otherwise we would have $K \in \mathcal{X}_{d}$ with $d=n / p_{1}^{\alpha_{1}}$.

Since all ideals of $K$ are principal one obtains the existence of $a \in \mathbb{Z}_{K}$ with $\left(a \mathbb{Z}_{K}, \mathfrak{f}\right)=1$ and $N(a) \not \equiv 1\left(\bmod p_{1}\right)$, as otherwise we would have $K \in \mathcal{X}_{p_{1}}$.

If $b \in \mathbb{Z}_{K}$ satisfies

$$
b \equiv a\left(\bmod p_{1}\right), \quad b \equiv 1\left(\bmod p_{2} p_{3} \cdots p_{s}\right),
$$

then

$$
N(b) \not \equiv 1\left(\bmod p_{1}\right), \quad N(b) \equiv 1\left(\bmod p_{i}\right), \quad i=2,3, \ldots, s,
$$

hence with $J=b \mathbb{Z}_{K}$ one gets $N(I J) \not \equiv 1\left(\bmod p_{1}\right)$ and

$$
N(I J) \equiv r_{j} \not \equiv 1\left(\bmod p_{j}\right)
$$

for $j=2,3, \ldots, s$, contradicting the Corollary 5 . 
TheOREM 3. (i) If $q_{1} \neq q_{2}$ are primes and $n=q_{1}^{a_{1}} q_{2}^{a_{2}}$, then $\mathcal{X}_{n}=\mathcal{Y}_{q_{1}} \cup \mathcal{Y}_{q_{2}}$.

(ii) If $K \in \mathcal{X}_{n}$ and the class-number of $K$ equals 1 , then $K \in \bigcup_{q \mid n} \mathcal{Y}_{q}, q$ ranging over prime divisors of $n$.

(iii) If $K \in \mathcal{X}_{n}$ is a Galois extension of the rationals and the fields $K$ and $Q\left(\zeta_{n}\right)$ are linearly disjoint, then $K \in \bigcup_{q \mid n} \mathcal{Y}_{q}$, q ranging over prime divisors of $n$.

Proof. (i) If $K \in \mathcal{X}_{q_{1} q_{2}} \backslash\left(\mathcal{Y}_{q_{1}} \cup \mathcal{Y}_{q_{2}}\right)$, then there are exist ideals $\mathfrak{p}_{1}, \mathfrak{p}_{2}$ not dividing $n \mathbb{Z}_{K}$ and satisfying

$$
N\left(\mathfrak{p}_{i}\right) \equiv 1\left(\bmod q_{i}\right), \quad i=1,2,
$$

and

$$
N\left(\mathfrak{p}_{1}\right) \not \equiv 1\left(\bmod q_{2}\right), \quad N\left(\mathfrak{p}_{2}\right) \equiv 1\left(\bmod q_{1}\right)
$$

Put $I=\mathfrak{p}_{1} \mathfrak{p}_{2}$ and observe that $N(I) \equiv N\left(\mathfrak{p}_{1}\right) N\left(\mathfrak{p}_{2}\right) \not \equiv 1\left(\bmod q_{i}\right)$ for $i=1,2$, contradicting Corollary 5.

(ii) Downward induction based on Lemma 4 shows the existence of a divisor $d$ of $n$ with $\omega(d) \leqslant 2$ and $K \in \mathcal{X}_{d}$. The assertion follows now in the case $d=1$ from Bauer's theorem, and in the case $d=2$ from (i).

(iii) It suffices to consider the case of $n$ square-free. Let $n=\prod_{j=1}^{s} q_{j}$ be the factorization of $n$ into primes, put $N=[K: Q]$ and for any divisor $d>1$ of $n$ denote by $K_{d}$ the field $K\left(\zeta_{d}\right)$. Since $K$ and $Q\left(\zeta_{n}\right)$ are linearly disjoint, the same applies to $K$ and $Q\left(\zeta_{d}\right)$, hence

$$
\left[K_{d}: Q\right]=[K: Q]\left[Q\left(\zeta_{d}\right): Q\right]=N \varphi(d)=N \prod_{q \mid d}(q-1)
$$

For any extension $k / Q$ denote by $P(k)$ the set of rational primes splitting in it, and let $d(k)$ be its density. Recall that if $k / Q$ is Galois, then $d(k)=1 /[k: Q]$ (see e.g. [6, Corollary 4 to Proposition 7.16]).

For $1<d \mid n$ denote by $X_{d}$ the set of rational primes splitting in $K / Q$ and satisfying $p \equiv$ $1(\bmod d)$ and observe that $X_{d}$ coincides with the set of rational primes splitting in both extensions $K / Q$ and $Q\left(\zeta_{d}\right) / Q$, hence $X_{d}=P\left(K_{d}\right)$.

The density of $P(K)$ equals $1 / N$, but using the equalities

$$
P(K)=\bigcup_{j=1}^{r} X_{q_{j}} \cup F
$$

with a finite set $F$, and

$$
d\left(K_{d}\right)=\frac{1}{\left[K_{d}: Q\right]}=\frac{1}{N \varphi(d)}
$$

and applying the inclusion-exclusion principle we get

$$
\frac{1}{N}=d(K)=-\sum_{1<d \mid n} \mu(d) d\left(K_{d}\right)=-\frac{1}{N} \sum_{1<d \mid n} \frac{\mu(d)}{\varphi(d)},
$$

hence

$$
0=1+\sum_{1<d \mid n} \frac{\mu(d)}{\varphi(d)}=\prod_{j=1}^{s}\left(1-\frac{1}{q_{j}-1}\right)
$$

and this shows that for a certain prime $q_{j} \mid n$ we have $q_{j}=2$, which is not possible, as $n$ is odd. 
6. We shall need a result of Pezda [7].

LEMma 5. If $R$ is the ring of integers in a finite extension of the p-adic field and $\mathfrak{P}$ is its unique maximal ideal, then the lengths of cycles in $R$ of polynomials from $R[X]$ are bounded by

$$
A(A-1) p^{1+\log _{2} e}
$$

where $A=\# R / \mathfrak{P}$ and $e$ is the ramification index, i.e. $p R=\mathfrak{P}^{e}$.

7. Proof of Theorem 2. We may assume that $K$ is totally complex, since if $K$ has a real conjugate $K^{\prime}$, then $F(X)$ is increasing on $K^{\prime}$, hence there are no $K^{\prime}$-cycles of length exceeding 1 , and the same applies to $K$.

Now we shall look for a prime ideal $\mathfrak{P}$ which does not divide $B$ and has its norm bounded by a constant, depending only on $n$ and the field $K$. By Theorem 1 every prime ideal dividing $B$ either divides $n$ or its norm is congruent to unity $\bmod q$ for a prime divisor $q$ of $n$. If all prime divisors of $n$ are larger than $2^{N}$, then prime ideals dividing $2 \mathbb{Z}_{K}$ cannot divide $B$, as their norms are $\leqslant 2^{N}$. If 2 splits in $K$, then any prime ideal dividing $2 \mathbb{Z}_{K}$ has norm 2 , and Theorem 1 implies the the cycles of $f$ in $K$ can have at most 2 elements.

If, however, $n$ has a prime ideal divisor of norm $\leqslant 2^{N}$, then we have to proceed in another way. The assumption $K \notin \mathcal{X}_{n}$ implies that there exists a prime ideal $\mathfrak{P}$ of $K$ with $(N(\mathfrak{P})(N(\mathfrak{P})-1), n)=$ 1 , and obviously its norm can be bounded by a constant depending only on $n$ and $K$.

Let now $L$ be the $\mathfrak{P}$-completion of the field $K$ and denote by $R$ its ring of integers. The choice of $\mathfrak{P}$ guarantees that our cycle lies in $R$ and $f \in R[X]$, hence we may apply Lemma 5 to get the assertions (i) and (ii) of the theorem.

Proof of Corollary 1. Assume that the extension $K / Q$ is Abelian and let $f$ be its conductor, i.e. $f$ is the minimal integer satisfying $K \subset Q\left(\zeta_{f}\right)$. If $H$ is the subgroup of the Galois group of $Q\left(\zeta_{f}\right)$ corresponding to $K$, then a prime $p$ splits in $K / Q$ if and only if $p(\bmod f)$ lies in $H$.

If $(f, M)=1$, then choose a prime $p$ satisfying

$$
p \equiv 1(\bmod f), \quad p \equiv-1(\bmod M) .
$$

Then $p$ splits in $K$ and satisfies $(p(p-1), n)=1$, hence prime ideals dividing of $p$ in $K$ do not divide $B$, and Linnik's theorem gives the assertion.

Now assume $(f, M)=d>1$. If $h \in H$ satisfies $h \equiv 1(\bmod q)$ for some prime $q \mid d$ and $p \equiv h(\bmod f)$, then $p \equiv 1(\bmod q)$. In view of $K \notin \mathcal{X}_{n}$ this cannot hold for all $h \in H$. Therefore there exists $h_{0} \in H$ such that for primes $q \mid d$ one has $h_{0} \not \equiv 1(\bmod q)$. Now let $D$ be the product of all prime divisors of $M$ not dividing $f$ and choose a prime $p$ satisfying

$$
p \equiv h_{0}(\bmod f), \quad p \equiv-1(\bmod D)
$$

Observe that $p$ does not divide $n$, then otherwise $p$ would divide $f$, hence $p \mid\left(f, h_{0}\right)=1$. Therefore $(p(p-1), n)=1$ and since $p$ splits in $K$, the preceding argument applies and we get the assertion.

Proof of Corollary 2. Apply Theorem 2 (i) and Theorem 3 (i).

Proof of Corollary 3. This follows from Theorem 2 (i) and Theorem 3 (ii).

Proof of Corollary 4. The condition $q-1 \nmid N$ implies the linear disjointness of $K$ and $Q\left(\zeta_{n}\right)$. If $K$ would belong to $\mathcal{X}_{n}$, then by Theorem 3 (iii) there would exist a prime divisor $q$ of $n$ with $K \in \mathcal{Y}_{q}$, hence $Q\left(\zeta_{q}\right) \subset K$ and $q-1 \mid N$, contrary to our assumption. It remains to apply Theorem 2 (i). 


\section{Bibliography}

[1] P. Morton, J. H. Silverman, "Rational periodic points of rational functions", Internat. Math. Res. Notices, 1994, № 2, 97-109.

[2] W. Narkiewicz, "Cycle-lengths of a class of monic binomials", Funct. Approx. Comment. Math., 42:2 (2010), 163-168.

[3] R. Walde, P. Russo, "Rational periodic points of the quadratic function $Q_{c}(x)=x^{2}+c$ ", Amer. Math. Monthly, 101:4 (1994), 318-331.

[4] G. S. Call, S. W. Goldstine, "Canonical heights on projective space", J. Number Theory, 63:2 (1997), 211-243.

[5] M. Bauer, "Zur Theorie der algebraischer Zahlkörper", Math. Ann., 77:3 (1916), 353-356.

[6] W. Narkiewicz, Elementary and Analytic Theory of Algebraic Numbers, 3rd ed., Springer Monogr. Math., Springer-Verlag, Berlin, 2004.

[7] T. Pezda, "Polynomial cycles in certain local domains", Acta Arith., 66:1 (1994), 11-22. 